\title{
Un silence numérique bavard. Controverses autour de l'interdiction de la propagande politique en ligne avant le vote
}

A chatty digital silence. Controversies over the banning of political propaganda prior to ballot day

Un silencio numérico vocinglero. Controversias alrededor de la prohibición de propaganda política en línea antes del voto

\section{Anaïs Theviot}

\section{(2) OpenEdition}

\section{Journals}

Édition électronique

URL : https://journals.openedition.org/mots/21482

DOI : $10.4000 /$ mots. 21482

ISSN : 1960-6001

Éditeur

ENS Éditions

Édition imprimée

Date de publication : 16 décembre 2013

Pagination : 55-70

ISBN : 978-2-84788-527-9

ISSN : 0243-6450

\section{Référence électronique}

Anaïs Theviot, « Un silence numérique bavard. Controverses autour de l'interdiction de la propagande politique en ligne avant le vote », Mots. Les langages du politique [En ligne], 103 | 2013, mis en ligne le 16 décembre 2015, consulté le 23 avril 2022. URL : http://journals.openedition.org/mots/21482 ; DOI : https://doi.org/10.4000/mots. 21482 


\title{
Un silence numérique bavard. Controverses autour de l'interdiction de la propagande politique en ligne avant le vote*
}

\begin{abstract}
Encore marginal au début des années deux mille, Internet s’est propulsé sur le devant de la scène politique française lors des campagnes sur le traité de 2005 établissant une Constitution pour l'Europe (Fouetillou, 2008) et lors de l'élection présidentielle de 2007 (Yanoshevsky, 2009). Cet attrait grandissant pour les technologies est particulièrement visible lors des campagnes électorales où les candidats se livrent à une véritable «course politique virtuelle» (Barboni, Treille, 2010, p.1137). La «campagne participative» de Ségolène Royal, avec le support de son association «Désirs d'avenir », s'est fortement appuyée sur des interfaces numérisées afin d'impliquer les militants et d'amorcer des débats en ligne (Beauvallet, 2007). Depuis cette élection présidentielle, les outils numériques se sont multipliés avec, notamment, l'arrivée massive des réseaux sociaux tels que Facebook ou Twitter.
\end{abstract}

À chaque débat télévisé réunissant un ou plusieurs candidats ou pendant les meetings, les «twittos " $^{1}$ sont présents pour rendre visible l'intervention de leur favori sur la Toile, les «fact-checkeurs $»^{2}$ détectent les incohérences de leurs adversaires et les «loleurs » 3 tentent de trouver un bon jeu de mots (PierreHenri Dumont, délégué national des Jeunes populaires : «\#flamby4 est en train de fondre sur place \#FranceMolle»). La bataille des tweets est devenue un enjeu pour les partis français pendant cette campagne; elle permet de mettre en scène une attention accrue à leur candidat et est souvent relayée par les

1. Utilisateurs de Twitter.

2. Personnes qui cherchent à vérifier les propos du candidat.

3. Tiré de l'anglais LOL (laughing outloud); désigne les internautes aux interventions humoristiques.

4. Il s'agit d'un sobriquet pour François Hollande. Ce surnom vient d'Arnaud Montebourg qui, en 2003, dans les couloirs de l'Assemblée nationale, fait cette comparaison : "Hollande, c'est Flamby. » Le dessert est connu pour son aspect flageolant, mais il reprend toujours sa forme initiale, même secoué dans tous les sens.

* Je tiens à remercier Antoine Roger, les évaluateurs de la revue et les coordinateurs de ce numéro pour leurs conseils.

Sciences po Bordeaux, Centre Émile Durkheim a.theviot@gmail.com 
médias traditionnels. La mobilisation en ligne offre également aux formations politiques le moyen de contourner les restrictions de temps de parole :

On avait tous compris que les nouvelles lois, les nouvelles régulations, qui seront à mon avis aussi les dernières, du CSA5 par exemple sur l'égalité absolue... ont favorisé Internet; c'est-à-dire que les grands médias sont devenus extrêmement ennuyeux, à cause de l'égalité absolue ; là pour le coup, l'activité sur internet s'est emballée. ${ }^{6}$

Toutefois, le vide juridique sur la Toile, en termes de régulation de la parole en ligne en période électorale, n'a pas duré. L'engouement pour les réseaux sociaux a ainsi engendré de nouvelles réglementations touchant également le domaine du numérique : la Commission nationale de contrôle de la campagne électorale (CNC) a ainsi précisé, dans le communiqué du 16 avril 2012, les règles en vigueur en matière de propagande électorale sur les nouveaux médias. Elle a demandé aux prétendants à l’Élysée « de ne plus diffuser par voie électronique aucun message ayant un caractère de propagande électorale à compter de vendredi à minuit». Cela comprend les sites Internet des candidats mais aussi les outils nouveaux, tels que Twitter et Facebook. Pendant les deux week-ends précédant le vote, le silence numérique est imposé aux partis politiques.

Quels sont les enjeux de ce silence numérique? Que recouvre cette expression? Comment cet «appel au silence» a-t-il été perçu par les «cyber-militants ${ }^{7}$ et les équipes web ${ }^{8}$ des partis politiques ? Comment les «états de gel» des sites et des réseaux sociaux ont-ils été anticipés?

Nous verrons qu'il est difficile de parler de silence au sens strict puisque celui-ci s'est révélé très «bavard » au sens où ce terme a suscité de nombreux débats au sein des instances politiques dans sa mise en application et a finalement été contourné par les cyber-militants.

Il conviendra dans un premier temps de questionner l'expression silence numérique : quel est le sens donné à cette formulation? Pourquoi parler de

5. Le Conseil supérieur de l'audiovisuel comptabilise les temps de parole des différents candidats pendant la durée de la campagne officielle.

6. Natalie Rastoin, directrice générale du groupe Ogilvy depuis janvier 2006, bénévole au sein de l'équipe web de François Hollande en charge de la réflexion stratégique (entretien du 15 septembre 2012).

7. Helen Margetts parle de «cyber-parti », au sens où le parti tendrait à se détacher des structures traditionnelles et des modes de communication en face-à-face et privilégierait la machine sans intermédiaire humain (Margetts, 2006). Nous reprenons ici sa formule en l'adossant cette fois-ci aux militants, mais dans une acception plus large : le cyber-militant serait un adhérent qui milite activement sur le Net. Pour en savoir plus sur le profil sociodémographique de ce cyber-militant, se référer à notre note de recherche (Theviot, à paraître).

8. Le Grand Dictionnaire terminologique de l'Office québécois de la langue française précise : «À l'origine, la graphie avec majuscule s'est imposée pour souligner le caractère unique du Web. On le considère alors comme un nom propre (tout comme le mot Internet d'ailleurs). Plus récemment, la graphie avec minuscule a fait son apparition dans la presse francophone et dans Internet. Signe que cet emprunt à l'anglais commence à s'intégrer à la langue française (on le traite comme un nom commun). » En somme, les deux formes, avec majuscules et minuscules, coexistent [http:// gdt.oqlf.gouv.qc.ca/ficheOqlf.aspx?Id_Fiche=2075076] (consulté le 26 juillet 2013). 


\begin{tabular}{|c|c|c|}
\hline $\begin{array}{l}\text { Semaine } \\
\text { du } 20 \text { avril } 2012\end{array}$ & $\begin{array}{c}\text { Facebook } \\
\text { (nombre de fans totalisé; } \\
\text { entre parenthèses, nombre } \\
\text { de fans gagnés dans la } \\
\text { semaine) }\end{array}$ & $\begin{array}{c}\text { Twitter } \\
\text { (nombre d'abonnés; entre } \\
\text { parenthèses, nombre } \\
\text { d'abonnés gagnés dans la } \\
\text { semaine) }\end{array}$ \\
\hline Parti socialiste (PS) & $44296(+1872)$ & $31508(+2300)$ \\
\hline $\begin{array}{l}\text { Union pour un mouvement } \\
\text { populaire (UMP) }\end{array}$ & $31789(+1980)$ & $28879(+2569)$ \\
\hline Parti radical & $662(+8)$ & $715(+31)$ \\
\hline $\begin{array}{l}\text { Mouvement démocrate } \\
\text { (MoDem) }\end{array}$ & $4959(+101)$ & $7057(+555)$ \\
\hline Europe Écologie - Les Verts & $17120(+150)$ & $80227(+1278)$ \\
\hline Nouveau Centre (NC) & $2166(+2)$ & $2178(+39)$ \\
\hline $\begin{array}{l}\text { Parti communiste français } \\
\text { (PCF) }\end{array}$ & $6596(+136)$ & $2751(+223)$ \\
\hline $\begin{array}{l}\text { Nouveau Parti anticapitaliste } \\
\text { (NPA) }\end{array}$ & $6619(+75)$ & $1889(+221)$ \\
\hline Front national (FN) & $69157(+1390)$ & $8824(+916)$ \\
\hline Parti de gauche (PG) & $8990(+743)$ & $7068(+663)$ \\
\hline République solidaire & $2925(-3)$ & $1083(-3)$ \\
\hline Lutte ouvrière & $75(+1)$ & $1242(+260)$ \\
\hline $\begin{array}{l}\text { Parti chrétien-démocrate } \\
\text { (PCD) }\end{array}$ & $1178(+7)$ & $492(+16)$ \\
\hline
\end{tabular}

Tableau 1. Présence des partis politiques sur les réseaux sociaux Facebook et Twitter 
silence alors que certains documents restent en ligne et qu'il est impossible de contrôler la parole de tous les internautes? Nous verrons que cette expression pose problème aux équipes de campagne des partis politiques (dans notre cas d'étude, le PS et l'UMP) car elle s'avère trop floue et ne peut être respectée dans une acception stricte sur la Toile. Nous procéderons ensuite à une analyse comparée des stratégies numériques adoptées, au PS et à l'UMP, pendant cette période de silence numérique imposé lors de la campagne présidentielle 2012 : l'anticipation de ce silence a engendré beaucoup de discussions au sein des équipes web du PS et de l'UMP afin de choisir les éléments les plus adaptés pour convaincre les indécis. Enfin, nous verrons que ce silence était en fait bien bavard, car les internautes ont réussi à contourner la règle en annonçant les résultats en amont sous forme de messages codés humoristiques.

Nos analyses s'appuient sur 35 entretiens 9 réalisés auprès des responsables numériques (et de la communication) de la campagne pour l'élection présidentielle française au PS et à l'UMP, une analyse de contenu du site Internet au moment de ce silence numérique, ainsi qu'une observation participante effectuée dans le cadre de notre recherche ${ }^{10}$, au sein de la direction du web du PS à Solférino du 1 ${ }^{\mathrm{er}}$ mars au 21 mai 2012.

\section{Silence numérique : une expression inappropriée et flottante}

Cette expression, silence numérique, a été employée dans le communiqué du 16 avril 2012 de la CNC afin d'expliciter les consignes à respecter par les partis politiques, en amont du premier tour de l'élection présidentielle française de 2012. Ce texte rappelle qu'à compter du vendredi 20 avril à minuit, la campagne électorale officielle prend fin et que «toute action de campagne et tout acte de propagande à visée électorale sont interdits sur l'ensemble du territoire de la République». L'interdiction de diffusion par voie électronique de messages à caractère de propagande électorale s'ajoute à l'interdiction de réunions publiques électorales et de distribution de tracts qui existaient déjà auparavant. La CNC a demandé aux candidats « de figer le contenu de leurs sites internet à partir de la même heure et de ne plus faire aucune actualisation de ces sites jusqu'à la fermeture des derniers bureaux de vote le dimanche 22 avril à 20 heures». Cette nouvelle disposition fait écho à celles qui concernent l'interdiction de publication des sondages en amont du scrutin (après la loi de 1977 et

9. Ce qui correspond à un échantillon important étant donné que les équipes web du PS, de l'UMP et du QG de Nicolas Sarkozy comptent respectivement une dizaine de membres et celle du QG de François Hollande autour de 40 personnes à la fin de la campagne.

10. «Militer sur Internet. Reconfigurations des stratégies partisanes et du militantisme au Parti socialiste et à l'Union pour un mouvement populaire pendant la campagne pour l'élection présidentielle française de 2012 ", thèse de science politique, sous la direction d'Antoine Roger, Sciences Po Bordeaux, Centre Émile Durkheim. 
la réduction du délai en 2002)11 qui est d'ailleurs mentionnée dans le communiqué : «Aucun sondage, aucun résultat partiel, aucune estimation des résultats ne peut non plus faire l'objet d'une quelconque diffusion le samedi 21 et le dimanche 22 avril avant 20 heures.»

Le syntagme silence numérique a ensuite été repris par les médias ${ }^{12}$, certains 13 lui préférant l'expression «état de gel » qui rend - il est vrai - davantage compte de l'immobilisme des pages web et profils Twitter ou Facebook des candidats. Cette formule concurrente souligne que l'emploi du terme silence est inapproprié dans son sens strict. Dans tous les cas - hors période de silence numérique -, lorsqu'on est en train de consulter le site de campagne des candidats, on se retrouve face au silence : rares sont les personnalités politiques qui accompagnent leur site de campagne d'un fond sonore, d'un hymne de campagne, de captation des sons d'ambiance d'un meeting par exemple... La mise en place de la webradio de François Hollande ${ }^{14}$ visait d'ailleurs à combler ce vide sonore en proposant aux internautes d'écouter en ligne une émission de radio donnant la parole aux équipes de campagne (pour suivre les coulisses de la campagne) et personnalités politiques. Il faut donc comprendre ce silence numérique dans une acception large du mot silence, dans la mesure où ce qui est ainsi désigné n'est pas l'interruption de la parole naturelle, mais la mise hors service des outils substitutifs de la voix humaine, qui permettent de relayer celle-ci. Il a en effet été demandé aux partis politiques de «désactiver tous les dispositifs d'actualisation automatique de leurs sites internet ainsi que tout dispositif permettant un échange interactif avec les électeurs, que ce soit par téléphone, par internet ou par voie d'échanges électroniques ». La formulation état de gel correspond bien à la première recommandation - pas de mise à jour des sites de campagne ou réseaux sociaux - tandis que le terme silence s'applique davantage à la seconde consigne - l'interruption des interactions. Encore faut-il qu'il existe bel et bien des interactions sur les sites de campagne des candidats. Plusieurs travaux ont déjà montré que, malgré des investissements importants dans le numérique, les partis politiques en période de campagne sont réticents à de véritables interactions permettant le dialogue avec les citoyens : l'étude comparative de Frédérick Bastien et Fabienne Greffet (2009, p. 213) souligne ce manque d’interactivité :

11. Après 1977 , les sondages d'opinion ne pouvaient plus être publiés pendant la semaine précédant chaque tour afin de préserver la liberté de choix des électeurs. Depuis la loi du 19 février 2002, les sondages peuvent être publiés jusqu'à la veille de chaque tour de scrutin.

12. "La Commission de contrôle veut un silence numérique des candidats à partir de vendredi » (Les Échos, 16 avril 2012); «2012: silence numérique à partir de samedi » (Europe1.fr, 16 avril 2012).

13. Les membres des équipes web interrogés préfèrent d'ailleurs employer l'expression état de gel, qui s'avère plus explicite et correspond davantage à la réalité puisque, nous le verrons, le silence est de toute façon déjà la règle sur les sites de campagne, ne disposant pas d'interface participative qui laisserait la parole aux internautes.

14. Première webradio créée en France à l'occasion d'une élection présidentielle, la station a été lancée le 26 mars et émettait du lundi au vendredi, de 18 heures à 19 h 30, depuis le QG de campagne de l'avenue de Ségur. 
Les partis incitent aussi leurs partisans à poser des gestes de militantisme en ligne, à occuper l'espace du web. [...] Cependant, les potentialités techniques du web 2.0, par exemple la coproduction de contenus par les usagers [...], n'étaient exploitées ni en France ni au Québec.

Les partis ont créé un genre hybride entre le web 1.0 et le web 2.0, résumé par l'expression «web 1.5 » de Jackson et Lilleker dans leur analyse de l'usage d'Internet par les partis politiques britanniques ${ }^{15}$. Selon Olivier Blondeau et Laurence Allard (2007, p. 45), les partis politiques feraient face à « des difficultés insurmontables » pour s'emparer réellement du réseau car ils ne sauraient se détacher de leur mode d'organisation traditionnel, à l'opposé de «l'esprit » d'Internet. Ainsi, les internautes sont déjà contraints au silence sur les sites de campagne de François Hollande et de Nicolas Sarkozy qui ne proposent pas d'espaces d'échanges. La dynamique participative - sujette à beaucoup de critiques $^{16}$ - n'a pas fait ses preuves lors de la campagne présidentielle française menée en 2007 par Ségolène Royal et n’a pas été réitérée en 2012 par le candidat socialiste, qui préfère "revenir aux fondamentaux» :

Ce que je retiens de la campagne de François Hollande en 2012, c'est que, finalement, ça a été une campagne assez traditionnelle. Ce que je veux dire, c'est que j'ai l'impression qu'on est revenu vraiment aux fondamentaux. Il n'y a pas eu vraiment, à mon avis, de rôle des nouvelles technologies, au sens large, dans la campagne, ça a été très marginal et c'est une campagne qui s'est d'abord jouée sur les grands médias. [...] Encore une fois, l’idée c'était vraiment de faire une campagne «président normal», de s'appuyer sur le parti tel qu'il était, etc. La démarche participative, ce qui n'est pas vraiment... C'était un truc très spécifique à Ségolène Royal mais ça ne fait pas partie des codes du Parti socialiste. Donc c'était un choix politique.17

L'injonction au silence - au niveau des internautes ${ }^{18}$ - fait davantage sens sur les réseaux sociaux que sur les sites de campagne (puisqu'il n'y a pas d'espaces participatifs proposés aux internautes). Toutefois, même sur Facebook, la parole est contrôlée : qu'il s'agisse du profil de François Hollande ou de celui de Nicolas Sarkozy, il n'y a pas de possibilité de laisser un commentaire, seuls les messages privés - qui ne s'affichent pas publiquement en ligne - sont possibles : «Des commentaires, non. C'était un choix. Parce qu'on n’avait pas de

15. "This reflects extensive use of the architecture of participation, but much less use of the community's democratic structure. This is clearly a hybrid or even bastardization of web 2.0 for promotional and marketing purposes. » (Jackson, Lilleker, 2009, p. 248)

16. La plateforme participative «Désirs d'avenir » a dû faire face à de nombreuses critiques, certains lui reprochant d'entretenir une illusion de prise en compte de la parole du militant lambda dans les processus de décision, en surfant sur la vague de la « démocratie participative» (Bousquet, 2007 ; Jullia, 2007).

17. Thomas Hollande, responsable du pôle vidéo au sein de la cellule numérique du QG de François Hollande (entretien du 10 octobre 2012).

18. Nous avons, dans notre recherche, deux approches : une "par le haut»- au niveau des institutions partisanes - et une «par le bas» - au niveau des adhérents et plus largement des militants actifs en ligne. 
ressources pour modérer et pas d'outils. „19 Si l'on s'en tient aux sites de campagne et comptes Facebook, l'expression «appeler au silence», alors que celuici existe déjà de fait, relève de l'hypocrisie.

En revanche, sur Twitter, la parole des internautes est libre et il n'est pas possible de bloquer les messages se référant aux candidats. Les partis politiques se trouvent donc dans une impossibilité technique, soumis aux règles de fonctionnement d'une interface qu'ils n'ont pas créée. Ils ne peuvent surveiller tous les partisans qui ne se tiendraient pas au silence et viendraient parler au nom du parti sur Twitter ou sur des espaces d'échanges en ligne tels que les forums de discussion. Cette imposition du silence est donc inappropriée et peu opérationnelle.

\section{Anticiper le silence en discutant}

Cette injonction au silence a fait l'objet de discussions en amont au sein des équipes web de campagne afin d'anticiper la paralysie des pages web et de contrôler la parole en ligne de leurs partisans. L'enjeu est, d'une part, de choisir un contenu de la page d'accueil du site de campagne du candidat qui soit le plus à même de convaincre les indécis qui vont s'y rendre le week-end précédant le vote; d'autre part, d'éviter que l'élection soit invalidée par la prise de parole de partisans allant contre la nouvelle réglementation. "La stratégie de gel des deux week-ends, c'est de savoir qui va venir sur les sites à ce moment-là : ce sont les indécis. Il faut avoir des choses très précises et très simples sur les raisons de voter François Hollande. On a toujours été dans le positif. » ${ }^{20} \mathrm{Au} \mathrm{QG}$ socialiste, cette réflexion a été menée au sein du comité stratégique de l'équipe numérique, composé de plusieurs responsables des différents pôles ${ }^{21}$ - contenu, influence, mobilisation, e-mails / base de données, atelier, veille et audiences - et des directrices opérationnelles :

On a monté ça [le comité stratégique] pour sentir les difficultés dans une campagne, prendre de la hauteur, alors que l'on est dans l'hyper-activité, et à gérer les urgences. Donc prendre de la hauteur par rapport à des sujets que potentiellement on pourrait anticiper. C'est-à-dire que là, la difficulté c'est qu'en campagne tu ne peux rien anticiper sauf quelques sujets comme notamment gérer les périodes de gel, entre vendredi minuit ou dimanche vingt heures. ${ }^{22}$

19. Axel Calandre, responsable des réseaux sociaux au sein de l'équipe numérique de Nicolas Sarkozy (entretien du 28 décembre 2012).

20. Claire Heuzé, coordinatrice mobilisation pour la campagne digitale de François Hollande (entretien du 4 juin 2012).

21. Appelés aussi «pétales» en référence à la rose socialiste.

22. Benjamin Fallot, responsable du pôle de veille au sein de l'équipe numérique de François Hollande et animateur du comité stratégique (entretien du 6 juin 2012). 
À l'UMP aussi, le gel du site internet de campagne a été pensé en amont, notamment à travers une sélection pointue des contenus devant apparaître en ligne pendant la période de silence numérique. Ces derniers devaient répondre aux critères suivants : clarté, concision, auxquelles s'ajoute le caractère percutant de l'argumentaire afin de marquer l'internaute de façon que son vote se porte sur le candidat promu en ligne :

C'était extrêmement réfléchi tout ça. II fallait que les choses qui étaient figées soient intéressantes et que les gens puissent consulter pendant le week-end. Donc là il y avait effectivement des contenus spécifiques. Il y avait les derniers argumentaires, en gros tout ce que l'on avait en réserve. Tous les trucs un peu lourds, on les mettait à ce moment-là. Dix bonnes raisons de voter Nicolas Sarkozy. Dix bonnes raisons de ne pas voter François Hollande. Là on sortait des argumentaires qui pouvaient être figés, mais que les gens pouvaient consulter. Il y avait un vrai travail. 23

Pendant plusieurs jours, les membres du comité stratégique, à droite comme à gauche, se sont réunis afin de réfléchir collectivement aux enjeux de cette nouvelle réglementation. Cette approche réflexive rompt avec la temporalité quasi instantanée du web : anticiper le silence a engendré de vrais débats sur la durée car les enjeux sont importants.

Quand on réclame le silence, on demande à l'assistance de se taire et d'éviter de faire du bruit. Le plus souvent, c'est pour faire mieux entendre un son qui se trouvait caché. Dans notre cas d'étude, il ne s'agit pas de son, mais l'effet est le même : figer une page internet permet de mettre en avant un contenu choisi. La difficulté pour les partis politiques est alors de savoir à qui ils s'adressent en priorité sur la page d'accueil : ceux qui connaissent déjà le site - les partisans ou ceux qui ont déjà consulté l'interface pour voter au premier tour - et ceux qui n'y ont jamais jeté un coup d'œil; ceux qui se portent traditionnellement vers l'extrême gauche ou ceux qui tendent vers l'extrême droite :

Plein de réunions stratégiques... entre les deux tours, tous les gens qui n'avaient pas voté pour l'un ou l'autre des candidats, une part non négligeable de ces électeurs, qui ne savaient pas quoi voter, s'est effectivement reportée sur les sites de candidats pour essayer de comprendre qui était... Donc, par exemple, pic de fréquentation, et là ça posait une question pédagogique, qui est : «ce sont des gens qui viennent comme des nouveaux, alors que nous on est déjà en fin de campagne, donc il faut refaire le contenu du site pour des nouveaux, parce que c'est des gens juste qui avaient voté Mélenchon qui n'étaient pas encore venus. Dans la manière de rééquilibrer les contenus sur le site, d'avoir des entrées directes, il fallait faire très attention à ne pas dégoûter et être peu accueillant envers les gens qui... alors que nous on était en fin de campagne, on avait fait toute notre histoire, ils venaient juste s'informer pour la première fois, etc. Donc ily a comme ça des tas de petits trucs, qui font une énorme différence... ${ }^{24}$

23. Olivier Gadan, membre de l'équipe web de Nicolas Sarkozy, en charge de la défense du bilan

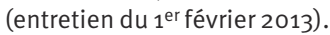

24. Natalie Rastoin, citée supra (entretien du 15 septembre 2012). 
L'emploi du terme silence a fait l'objet de controverses au sein des directions des équipes de campagnes des partis politiques puisque, employé dans une acception large, il peut être interprété de manière très différente. Ce terme flottant engendre ainsi des interrogations sur les périmètres de son application : « Nous sommes en train de contacter plusieurs soutiens mais la question est de connaître précisément la limite du périmètre. »25

La loi est pas claire. La loi est pas claire et y avait énormément de problèmes d'interprétation entre même nos conseillers juridiques pour dire est-ce que par exemple un twittos qui porte les couleurs de François Hollande, est-ce que s’il fait un... il diffuse l'information interdite, est-ce que ça impacte sur la campagne de François Hollande? Et la réponse n'était pas claire. Y a des avocats qui nous ont dit «mais oui attention, il peut être après considéré comme étant membre de votre équipe de campagne ». Donc c'était un merdier sans nom. Donc nous, on était assez inquiets là-dessus et on avait à la fois... on avait vraiment passé l'information en amont, on avait fait plein de mailings à ce sujet-là : «Attention, arrêtez de twitter, arrêtez de Facebooker, plus de blog, plus rien à partir de minuit». Nous-mêmes, on avait mis en place une équipe, une cellule de veille spéciale pendant le week-end pour à la fois, alors pas tant surveiller les erreurs de notre côté que surveiller et faire... prendre des preuves de toutes les erreurs de la droite pour si jamais... moi j'avais dit «si jamais après y a un contentieux juridique... $»^{26}$

Des questions stratégiques se jouent là, au regard de l'application que vont en faire les autres partis politiques : fermer la page Facebook, la laisser ouverte mais bloquer les messages privés, surveiller la parole en ligne de ses partisans ou les laisser libres de leurs propos puisqu'ils ne font pas officiellement partie de l'équipe de campagne... D'autant plus que cette nouvelle réglementation s'applique dans un contexte de confrontation partisane, ce qui pousse les partis politiques à se mettre davantage en avant que leurs concurrents, à montrer qu'ils ont mieux respecté la consigne que les autres, dans l'objectif notamment de «jouer le bon élève » médiatiquement : «On a désactivé le site toushollande 27 . On a été très stricts dans le respect des consignes, on a désactivé tous les boutons de partage, ce que n'a pas fait l'UMP » ${ }^{28}$; "On avait même désactivé le profil Facebook et tout fermé pour ne prendre vraiment aucun risque ${ }^{29}$. Avant l'heure fatidique de dernière mise à jour autorisée des sites de campagne, les partis se surveillent mutuellement pour savoir ce que le concurrent a choisi de mettre en avant sur sa page d'accueil : «C'était génial. On s’est amusé. On a

25. Nicolas Princen, directeur du web au QG de Nicolas Sarkozy (entretien du 15 septembre 2012).

26. Romain Pigenel, responsable de la cellule «Influence» au QG de François Hollande (entretien du 11 octobre 2012).

27. Il s’agit du site de campagne dédié à la mobilisation en ligne géré par l'équipe numérique de François Hollande.

28. Claire Heuzé, citée supra (entretien du 4 juin 2012).

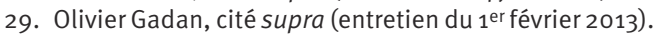


vraiment mis en ligne le site genre à 23 h 50 ! Au premier comme au deuxième tour! Il y avait ce côté un peu marrant, “Bon, ils vont sortir quoi ?” „30

Il est intéressant de souligner que ces débats, engendrés par la nouvelle réglementation et l'emploi du terme silence, se sont circonscrits aux membres de l'équipe web et n'ont pas été pensés dans un plan de communication global, ce qui est assez significatif de la faible intégration du web dans la campagne et de la place qui lui est accordée :

Comme Manuel Valls n'avait pas la main sur le numérique, il contrôle ce qu'il contrôle. Ce qu'il ne contrôle pas ça ne l'intéresse pas. La campagne web, ce n'était pas lui. Il y a d'un côté la com qui a beaucoup plus de puissance. La com des chaînes d'info et de la presse, ça c'est Manuel. [...] Et puis il y avait l'équipe web. [...] On avait fini la mise en ligne et ça s'était fait sans Vincent Feltesse, et les deux fois [les deux fois précédant le silence numérique] ! Sans personne de l’État! Personne! Pour eux ce n'était pas un enjeu !31

\section{Un silence numérique contourné par l'humour}

Cette contrainte pesant sur le média - paralysie des outils web de campagne est subjectivement vécue comme un appel au silence par ceux à qui elle est imposée. Les partis politiques se sentent ainsi dans l'obligation de faire passer cette consigne auprès de leurs partisans les plus actifs en ligne. Au QG de François Hollande a été mise en place une équipe chargée d'une veille en ligne afin de surveiller la parole de ses partisans et de leur demander de «se taire» - de ne plus twitter ni mettre de message sur leurs comptes personnels en faveur du candidat. Ce dispositif en interne vient compléter celui instauré par le CNC qui a chargé une dizaine de personnes d'assurer la surveillance des réseaux sociaux et du web. Mais il s'avère très périlleux de faire régner le silence sur la Toile. La culture Internet va à l'encontre de la notion de «contrôle» de la parole. Imposer le silence est vécu par les internautes militants comme une forme de censure et fait écho à un engagement libéré et plus distancié32. Employer le terme silence alors que dans les textes, il ne s'agit que d'un état de gel des pages web / réseaux sociaux du candidat, contribue à mettre le feu aux poudres. La libre circulation de l'information et des opinions sur la Toile est l'essence des discussions en ligne. Même s’il s’agit souvent de «monologues interactifs» (Dumoulin, 2002, p. 156),

30. Lionel Bordeaux, rédacteur en chef du site françoishoallande.fr / webradio et responsable du pôle "contenu » au sein du QG de François Hollande (entretien du 2 janvier 2013).

31. Ibid.

32. La personne engagée semble avoir de plus en plus besoin de s'exprimer librement. Le parti ou l'association sont alors perçus comme des supports à la diffusion de ses convictions et non comme un carcan. Comme le soulignent Anne Quéniart et Julie Jacques (2001, p. 48), «ce refus d'une certaine unification de la pensée, ce besoin de liberté de parole, cette absence, pourrait-on dire, de fidélité absolue au parti, tout cela traduit bien les changements de sens de l'engagement politique» (nous soulignons). 
c'est-à-dire de messages qui forment une unité sans remise en cause de leur propre opinion, les internautes refusent de se sentir contraints par une législation. Gersende Blanchard, dans son analyse des discussions sur les forums des sites internet des partis politiques français hors période de campagne, souligne que la parole critique est abondante - elle va même jusqu'à parler de «règne de la critique» (Blanchard, 2007). En période de campagne, il s'agit davantage de soutenir son favori ou d'engager un débat sur les propositions, comme a pu le montrer Nicolas Desquinabo, durant la campagne présidentielle française de 2007, dans son étude des espaces de discussion proposés par trois principaux partis politiques - UDF, UMP et PS (Desquinabo, 2011). Se contraindre au silence 48 heures avant le moment crucial des résultats est un impensable pour des militants qui ont été en permanence sollicités par le parti pour militer en ligne33.

La diffusion de résultats d'élections générales, même partiels, y compris par voie électronique, est interdite avant la fermeture du dernier bureau de vote à 20 heures et passible d'une amende pouvant aller jusqu'à 75000 euros (article L52-2 du code électoral). En 2007, déjà, les estimations des instituts de sondage avaient été relayées, notamment par des sites internet belges et suisses. En 2012, c'est sur Twitter que les internautes et même les personnalités politiques - pourtant légalement contraintes au silence - ont révélé en premier le résultat de l'élection présidentielle. Pour éviter de devoir payer une amende, la plupart des twittos se sont amusés à trouver des messages codés, souvent sur le ton humoristique. Stéphanie Wojcik, dans son analyse des forums municipaux, avait mis en évidence l'usage de l'humour dans les critiques les plus sévères envers les autorités municipales (2005, p.344). En effet, l'humour est un registre courant sur la Toile pour faire passer des messages critiques ou à la marge (ici illégaux), comme nous avions déjà pu le montrer dans notre analyse des messages postés sur le réseau social socialiste, la Coopol (Theviot, 2012). Par exemple, on trouve des bulletins météorologiques qui font référence aux candidats pour la ville et au pourcentage des votes pour la température : «Premières prévisions METEO pour ce soir - Corrèze 28?, Neuilly 25 ?, Nuremberg 15 ?, Moscou 14 ?, Bearn [sic] 10 ?, Norvège 3 ? \#RadioLondres. » Les jeux de mots autour du nom de famille du candidat du PS et du pays d'origine de la famille de son rival de l'UMP constituent également des codes récurrents dans les messages des twittos: «\#RadioLondres une grosse vague des Pays-Bas est en train de s'abattre sur la Hongrie» ou encore «Le gouda sent bon chez les Belges, je répète le gouda sent bon chez les Belges

33. Déjà, en 2011, lors de l'émission «Paroles de Français» (où Nicolas Sarkozy dialoguait avec neuf citoyens) diffusée sur TF1 le 10 février, Jean-François Copé, secrétaire général de l’UMP, avait envoyé un mail à l'ensemble des militants pour les inciter à « relayer les messages » du président: «Nous serons unis pour faire face aux campagnes de désinformation et de démagogie orchestrées par l'opposition et relayées sans scrupule par certains médias. Je compte particulièrement sur vous pour faire part de vos réactions et commentaires sur les différents sites d'actualités disponibles sur Internet. » (Extrait du mail envoyé par Jean-François Copé) 
\#radiolondres \#France2012 ». Les surnoms - peu flatteurs - des candidats tels que «Flamby » pour François Hollande et «talonnettes» ou « Rolex» pour Nicolas Sarkozy se retrouvent également dans les tweets codés : «Martinique : $51 \%$ Flamby, Talonnette $26 \%$ Papon 4,76\% \#radiolondres»; «Ici \#radiolondres le flamby se démoule à 28 degrés »; "Rolex au poignet, un second tour à Amsterdam. \#RadioLondres». Les exemples donnés ci-dessus relèvent davantage du registre de l'ironie ou de la dérision car, comme le souligne Arnaud Mercier (2001, p.10), «tourner en ridicule, mépriser, souligner l'insignifiance, tels sont les traits associés à la dérision et qui permettent de la distinguer des notions de rire et de comique (termes généraux) ou d'humour (aux intentions déstabilisatrices beaucoup moins affirmées) ». L'emploi des sobriquets vise dans un premier mouvement à contourner la règle du silence, mais aussi à tourner en ridicule la figure des dirigeants, en forçant sciemment le trait pour souligner l'absurdité d'une situation donnée.

Des internautes indiquent, pour le non-initié à Twitter, où trouver l'information : «\#radiolondres rtbf. de pour les results DOM-TOM, je dis ça je dis rien... ». En effet, lors des soirées électorales, c'est le hashtag (mot clé) \#RadioLondres qui a été utilisé pour répertorier les résultats du scrutin, faisant bien entendu écho à la BBC qui, en 1940, avait ouvert ses ondes aux premiers porte-parole de la France libre.

Ben, ça n'a pas marché. Ils sont exposés. C'est-à-dire qu'à droite, à gauche, y a eu énormément, énormément d'infractions à la loi. Parce que la loi est plus adaptée, parce que la loi elle est... elle est prévue pour un paradigme où en gros vous avez d'un côté les médias, de l'autre côté la population. Or, le principe même de Twitter et des blogs, c'est que chaque personne devient son propre média. Donc c'est impossible. Autant vous pouvez dealer avec les rédactions presse de se taire jusqu'au moment du scrutin mais c'est impossible... alors nous, on avait fait passer énormément de messages en amont «arrêtez de twitter ». [...] Et ça a été catastrophique. C'est-à-dire que les informations ont fuité partout, des élus, à droite comme à gauche, y a des comptes Twitter qui n'avaient rien compris à la loi, ils faisaient encore des tweets de mobilisation ou des posts de blogs dans le week-end. Enfin c'était n'importe quoi. Du coup, personne n'a rien dit, mais c'est vrai que dans ces cas-là, faut en tirer les conclusions, c'est-à-dire que la loi n'est plus adaptée. Bon, au début moi, j'étais extrêmement inquiet et puis après quand j'ai vu que ça explosait dans tous les sens, y aura rien derrière. On peut pas faire invalider une élection là-dessus. 34

Ce contournement de la règle s'est fait de façon si massive sur le réseau, à droite comme à gauche, qu'il n'a pas été possible d'entamer des poursuites judiciaires pour sanctionner les twittos et que la menace d'une invalidation de l’élection n'a pu être formulée.

34. Romain Pigenel, responsable de la cellule «Influence» au QG de François Hollande (entretien du 11 octobre 2012). 
L'objectif de cet article était de réfléchir aux contours donnés à la notion de silence dans la nouvelle réglementation mise en place pour limiter la propagande partisane en ligne avant le vote : que recouvre cette expression? Comment les équipes web l'ont-elles comprise et appliquée? Il en ressort que ce syntagme, silence numérique, s'avère inadapté et flottant. Controversée au sein des instances partisanes, cette notion floue a contraint les équipes web à élaborer une définition subjective et pragmatique afin d'identifier comment appliquer, concrètement, sur leurs supports numériques de campagne, la loi. Cette réflexion avait déjà pu être esquissée, de manière non contrainte, à l'UMP lors de l'affaire Mehra puisque ce parti politique avait décidé de «suspendre» l'activité de son site de campagne :

Vous avez eu plusieurs silences numériques. Vous avez eu l'affaire Mehra déjà, où on a fermé le site pendant trois ou quatre jours. [...] On avait mis une page de deuil et on ne touchait plus à rien. [...] On n'a pas pour autant rien fait pendant cette période-là. On a préparé d'autres choses. On a avancé sur d'autres dossiers. Et surtout on s'interrogeait sur ce que l'on mettrait quand le site rouvrirait parce que c'est toujours un peu difficile cette transition. Ensuite vous avez des périodes de silence numérique qui sont imposées par la loi électorale. 35

À droite comme à gauche, l'imposition du silence par l'État (CNN) a engendré de nombreux débats stratégiques (PS/UMP), si bien que l'on peut parler d'un «silence numérique bavard». D’autant plus que les internautes ne se sont pas sentis concernés par cette loi - bien que les adhérents fortement impliqués et les élus aient été avertis par leur parti - et ne se sont pas privés de prendre part aux conversations politiques en ligne dans les 48 heures avant l'élection. L'arrêt de l'activité militante avant les résultats existait déjà avec la législation sur l'interdiction de tracter, mais cette activité ne s'inscrit pas dans la même temporalité que la militance en ligne. Poster un message sur Twitter relève de l'instantané et certains partisans ont pris l'habitude, pendant la campagne, de s'exprimer des dizaines de fois par jour via ce dispositif; si bien qu'ils ne peuvent s'en passer. S'imposer le silence, c'est ne plus être acteur de la victoire de son favori. Le silence est alors oppressant car les partisans ne peuvent plus se sentir investis d'un rôle pouvant faire pencher la balance à travers des échanges d'argumentations sur la Toile. Cette quasi-addiction et la volonté d'agir pour la victoire jusqu'au dernier moment les ont amenés à contourner, à jouer avec la règle, à «braconner, ruser, inventer» (De Certeau, 1980, p. 174) pour continuer à s'exprimer en ligne. Dans cet article, nous avons déjà repéré que l'humour s'avère une arme discursive pour les internautes souhaitant contourner la loi

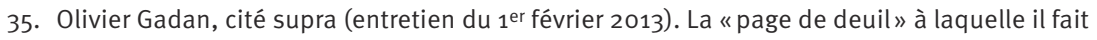
allusion présente, dans un rectangle orné un bandeau noir, une phrase de Nicolas Sarkozy, en blanc sur fond bleu : «Face à la tragédie nationale que nous vivons, je suspends ma participation à la campagne présidentielle au moins jusqu’à mercredi. » 
et dévoiler les résultats de l'élection présidentielle. Mais, au-delà de cet enjeu, savoir ce qui se dit en ligne dans les premières 24 heures précédant le vote (et non les dernières 24 heures correspondant davantage à l'annonce des résultats), c'est-à-dire lorsque les internautes peuvent encore convaincre certains indécis en ligne, pourrait nous apporter des pistes de réflexion sur le rôle joué par les dispositifs numériques et sur l'impact de l'activisme en ligne. L'idée est de s'intéresser à ce que disent les adhérents en ligne au moment crucial de l'avantvote; alors que leurs cadres politiques ne peuvent plus s'exprimer, contraints au silence numérique. Est-ce un moment d'émancipation de l'internaute, une affirmation de son identité militante personnelle face à «l'autoritarisme » de l'institution, qu'elle soit étatique (CNC) ou politique (PS/UMP) ? Ou le cyber-militant est-il un «bon soldat» de l'institution partisane agissant toujours en supporter de son candidat - même quand il est le seul à pouvoir s'exprimer - et trouvant la rétribution de son militantisme dans sa victoire?

Encore faut-il être certain que les responsables partisans - y compris les membres de l'équipe de campagne numérique - ne contournent pas la règle. Après la victoire de François Hollande, l'interview de son directeur artistique, publié en ligne dans un site spécialisé sur la web campagne, a fait le «buzz» au sein du microcosme politique. Celui-ci explique :

Je diffusais mes photo-montages depuis mon compte Facebook personnel, pour que ça ne paraisse pas officiel. Les images et les vidéos se répandaient ensuite toutes seules sur YouTube et sur les réseaux sociaux. Elles restent introuvables sur les pages Facebook et les comptes Twitter officiels du candidat. ${ }^{36}$

Robin King déclare alors être à l'origine du photomontage Marine Le Pen / Nicolas Sarkozy37 - détournement d'une affiche de la marque de vêtement «The Kooples»-diffusé sur son «mur»38 Facebook, le 26 avril 2012, et partagé ensuite par près d'un millier d'internautes. À partir de cet exemple, nous pouvons imaginer que les équipes web de campagne de l'UMP et du PS ont mis en place des stratégies officieuses (non évoquées lors des entretiens semi-directifs) de contournement du silence numérique imposé au moment crucial de l'avantvote. Ce type de «jeu avec la règle »39 et le travail de destruction de la « e-réputa-

36. Robin King, directeur artistique et membre de l'équipe web de François Hollande. Extrait de l'interview accordé à la webcampagne.fr, mis en ligne le 15 mai 2012 [http://www. lawebcampagne.fr/?p=388]. Consulté le 26 juillet 2013.

37. . Les deux candidats y apparaissent côte à côte, proches l'un de l'autre, tandis que sous la marque «The Kooples», qui barre l'image, figure cette légende : «Marine \& Nicolas en couple depuis 5 ans».

38. Un «mur» (wall en anglais) est la dénomination employée sur le réseau social Facebook pour désigner la page où les amis et l'utilisateur peuvent écrire des messages de façon publique, c'est-à-dire que tous les internautes peuvent les lire (cela dépend encore des paramètres de confidentialité choisis par l'utilisateur qui, le plus souvent, réserve cette fonction uniquement à ses amis).

39. Les équipes de campagne de Barack Obama seraient à l’origine de nombreux «faux» comptes 
tion ${ }^{40}$ de l'adversaire rappellent les campagnes américaines. En 2012, l'équipe de Barack Obama a adopté une stratégie d'attaques contre Mitt Romney, visant en particulier sa carrière dans le capital-risque. Il est dépeint dans des vidéos en ligne comme un «vampire», un «destructeur d'emplois» ou le «contraire de Robin des Bois»; et à la fin de ces films officiels, conformément à la loi américaine, on entend le président dire : «Je suis Barack Obama, et j’approuve ce message. » Pour prolonger cette enquête, il serait pertinent d'adopter une démarche comparative avec nos voisins américains afin de mettre en perspective les différentes cultures (partisanes) et leurs influences sur la mise en place de réglementation (ou non) dans les campagnes en ligne.

\section{Références}

BARBONI Thierry, TREILLE Éric, 2010, "L'engagement 2.0», Revue française de science politique, vol.60, n6, p.1137-1157.

BASTIEN Frédérick, GREFFET Fabienne, 2009, "Les campagnes électorales à l'ère d'Internet. Une comparaison des sites partisans en France et au Québec», Hermès, $\mathrm{n}^{\circ}$ 54. Également paru dans Internet et politique, A. Coutant éd., "Les Essentiels d'Hermès», Paris, CNRS, 2012.

Beauvallet Godefroy, 2007, «Partie de campagne. Militer en ligne au sein de "Désirs d'avenir" ", Hermès, nº 47, p. 27-40.

BLANCHARD Gersende, 2007, "La communication politique partisane sur Internet. Des pratiques et des stratégies nouvelles? ", thèse de doctorat en sciences de l'information et de la communication, sous la dir. de I. Pailliart, Gresec, Université Stendhal Grenoble 3.

Blondeau Oivier, Allard Laurence, 2007, Devenir média. L'activisme sur Internet, entre défection et expérimentation, Paris, Éditions Amsterdam.

BOUSQUET Franck, 2007, «L'Internet collaboratif est-il participatif? Le blog de DSK et Désirs d'avenir durant la pré-campagne de désignation du candidat socialiste à l'élection présidentielle», Colloque «Les usages partisans de l’Internet», 21-22 juin 2007, Nancy.

De Certeau Michel, 1980, L'invention du quotidien, t. I, Arts de faire, Paris, Gallimard.

DESQUINABO Nicolas, 2011, «Polémique ou délibération? Les usages des forums partisans durant la campagne de 2007 ", Continuerlalutte.com. Les partis politiques sur le web, Paris, Presses de Sciences Po, p. 249-265.

Dumoulın Michaël, 2002, «Les forums électroniques. Délibératifs et démocratiques?»,

Twitter parodiques et agressifs ayant pour but de propager en ligne une image négative de l'adversaire: [http://140elect.com/twitter-politics/100-rules-of-effective-political-tweeting/]. Consulté le 26 juillet 2013.

40. Se référer à "E-réputation et traces numériques. Dimensions instrumentales et enjeux de société», et notamment l'atelier 3 : "Les dimensions instrumentales de l'e-réputation », colloque international organisé par l'équipe Médias, culture et communication (MC2) de l'Idetcom, Université Toulouse 1 Capitole, 21 et 22 mars 2013. 
Internet et la démocratie. Les usages politiques d'Internet en France, au Canada et aux États-Unis, D. Monière éd., Québec, Monière et Wollank, p. 141-157.

Fouetillou Guilhem, 2008, «Le web et le traité constitutionnel européen », Réseaux, n०147, p. 229-257.

JACKSON Nigel A., LILleKER Darren G., 2011, «Microblogging, constituency service and impression management. UK MPs and the use of Twitter», The Journal of Legislative Studies, vol. 17, nº1, p. 86-105.

JULlıA Patricia, 2007, «Les enjeux de l'expression politique en ligne entre mythes et réalités. Démocratie participative, café du commerce et nouvelle propagande », Colloque "Les usages partisans de l’Internet », 21-22 juin 2007, Nancy.

MARgetts Helen, 2006, «Cyber Parties», Handbook of Party Politics, R. Katz, W. Crotty éd., Londres, Sage, p.528-535.

Mercier Arnaud, 2001, «Pouvoirs de la dérision, dérision des pouvoirs », Hermès, nº 29, p. 9-18.

QUENIART Anne, JACQUES Julie, 2001, "L'engagement politique des jeunes femmes au Québec. De la responsabilité au pouvoir d'agir pour un changement de société », Lien social et Politiques, $n^{\circ} 46$, p. 45-53.

THEVıOT Anaïs, à paraître (2013), «Qui milite sur Internet? Esquisse du profil sociologique du “cyber-militant” au PS et à l'UMP», Revue française de science politique.

— 2012, "Militer tout en critiquant publiquement son parti. Le cas du réseau social socialiste, la Coopol », Cahiers du Centre Émile Durkheim, nº12, p.1-44 [http://centredurkheim.fr/Cahiers\%20du\%20CED_12/Cahiers\%20du\%20CED_12.pdf]. Consulté le 26 juillet 2013.

Wojcık Stéphanie, 2010, Délibération électronique et démocratie locale. Le cas des forums municipaux des régions Aquitaine, Languedoc-Roussillon et Midi-Pyrénées, Sarrebrück, Éditions universitaires européennes, 2010 (thèse de doctorat de science politique soutenue en 2005, Université Toulouse 1).

YANOSHEVSKY Galia, 2009, "L'usage des vidéoblogs dans l'élection présidentielle de 2007. Vers une image plurigérée des candidats», Mots. Les langages du politique, n89, Débats pourl'Élysée, D. Barbet, D. Mayaffre éd., p. 57-68. 